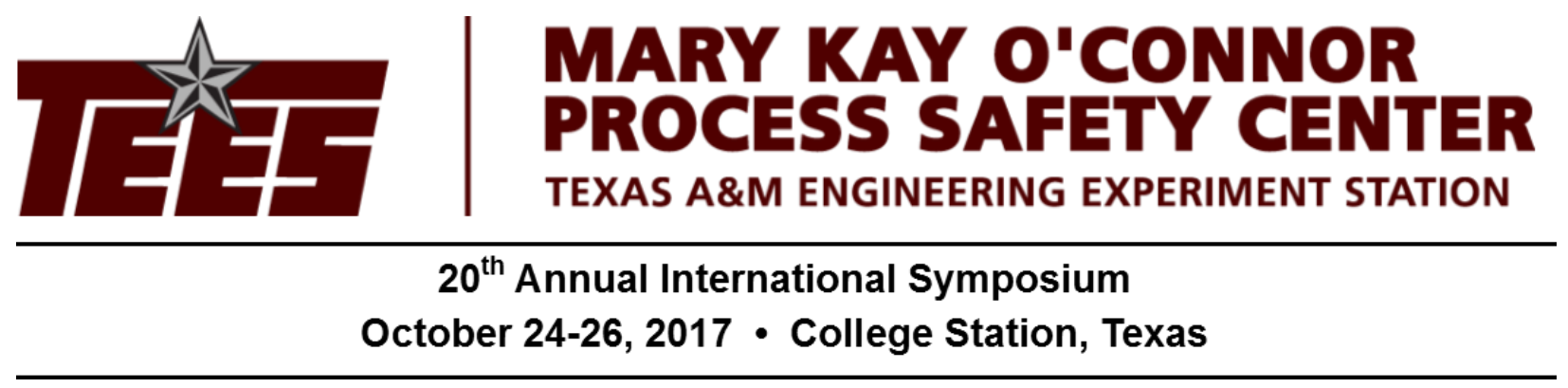

\title{
Improved Blast Capacity of Pre-engineered Metal Buildings using Coupled CFD and FEA Modeling
}

\author{
James Wesevich, D. Milner, L. Nikodym, V. Nasri, D. Lawver, J. Mould \\ Thornton Tomasetti, Weidlinger Applied Science \\ 115 Wild Basin Road, Suite 309 \\ Austin, Texas 78746, USA \\ JWesevich@ThorntonTomasetti.com
}

\begin{abstract}
The initial CCPS guideline [CCPS, 1996] for estimating lethality for building occupants within petrochemical buildings subjected to blast hazards was based on building construction type and peak overpressure. This method allows for a quick screening of building occupant vulnerability but does not include the effects of the duration of the blast that the buildings are subjected to. Blast hazards within petrochemical facilities include vapor cloud explosions (VCE), BLEVEs, and bursting pressure vessels. VCE's include both deflagrations having long blast durations and detonations having much shorter durations. Bursting pressure vessels can also be characterized as having relatively short durations. Therefore, the latest CCPS guideline [CCPS, 2012] removed this singular peak overpressure methodology and provided occupant vulnerability as a function of building damage and construction type as a function of peak overpressure and duration. Unfortunately, this improved method on blast characterization did not provide a way to correlate blast loading with building damage.
\end{abstract}

This blast-to-damage correlation is required to conduct quantitative risk assessments. A range of simplified tools are available for assessing the response of structural components and building construction types to blast loads. These tools include Single Degree of Freedom (SDOF) models and Pressure-Impulse (P-I) iso-damage charts. These simplified tools generally do not account for the complex response and failure of real structures or the difference in response to different forms of blast loading that include finite rise times (blast waves). Iso-damage charts may be based upon historical data gathered from a range of sources and are often based upon blast damage caused by High Explosive (HE) detonations.

This presentation illustrates the use of multi-degree of freedom structural systems for a preengineered metal building (PEMB). PEMBs represent the majority of building construction within petrochemical facilities. Computational Structural Dynamic (CSD) finite element analysis (FEA) and Computational Fluid Dynamic (CFD) approaches are used to show the level of conservatism 
in estimating the blast capacity of PEMBs compared to more traditional SDOF methods. Fully coupled CFD and FEA modeling that includes the beneficial effect of including the air that is internal to the building is demonstrated.

\section{Key Words}

Petrochemical facility siting, VCE, CSD, CFD, FEA, blast pressure and impulse, blast waveform, PEMB

\section{Introduction}

Current methods of predicting structural damage in pre-engineered metal buildings (PEMBs) subject to vapor cloud explosions are quick and cost-effective to perform. However, they also tend to be overly conservative; costs saved on analysis may be more than offset by expensive retrofits or other mitigation measures. This paper investigates the effectiveness of using more advanced computational techniques with increasing levels of refinement and sophistication to produce more realistic estimates of structural damage and corresponding human injury. The goal is to reduce the amount of over-conservatism in results and avoid the degree of costly mitigation measures that may be unnecessary.

In this study, a typical PEMB is selected and assessed for selected loads using a variety of approaches that ramp up in the level of analytical sophistication. The extent of damage is determined by each method and the results are compared to see the benefit of each analytical method.

\section{Description of the PEMB Studied}

Metal building systems were pioneered in the early 1900's. One of the first companies, STEELOX, developed an interlocking metal panel that was displayed at the Century of Progress Exposition in 1934. Over the next several decades, the design of these systems was optimized and standardized throughout the industry. Metal building systems use cold-formed panels of sheet metal for wall and roof cladding. The panels are supported by girts and purlins made of light gauge cold-formed Z- and C-sections, which are in turn supported by structural steel frames using hot-rolled built-up, tapered members. The sizes of the light gauge and structural steel sections vary with building spans and loading. In the decades since their introduction, the designs of these buildings have been optimized and essentially standardized, including lighter steel thicknesses and higher steel strengths.

The structure analyzed here is based on an actual building designed by a major PEMB manufacturer. It measures $60 \mathrm{ft} \times 100 \mathrm{ft}$ in plan, with an eave height of $25 \mathrm{ft}$ and ridge height of $30 \mathrm{ft}$. Moment frames span in the $60 \mathrm{ft}$ direction. Their spacing formed five bays of approximately $20 \mathrm{ft}$ each. The short exterior ends were comprised of W10 and W12 steel columns/wind posts that divide each face into three bays. The columns are placed to accommodate doors of various sizes and placements; one side of the structure has three 
approximately equal bays of $20 \mathrm{ft}$, while bays on the opposite face are approximately $17 \mathrm{ft}, 20 \mathrm{ft}$, and $23 \mathrm{ft}$.

The steel framing supports girts and purlins formed of sheet metal. All girts and purlins use Zshaped 8 in deep cross-sections with approximately 3 in long flanges. They are formed from 12, 14 , and 16 gauge sheet metal, and are typically connected to the structural steel framing with two $1 / 2$ in diameter bolts. Girts are typically spaced at 44 in to 51 in on center, while purlins are spaced at 44 in on center.

The sides of the building are clad with 24 gauge sheet metal. Each panel is 36 in wide and features corrugations 1.25 in high. The roof is clad with 24 gauge sheet metal panels, each 16 in wide with 2 in standing seams. Both wall and roof cladding are attached to the girts and purlins by self-tapping screws.

Structural models and analyses were based on fabrication drawings and thus reflect some of the variations in framing typical of actual PEMB structures. In order to facilitate ease of modeling and speed of analysis, however, some simplifications and modifications were made to the structure. First, the structure was assumed to be symmetric with a plane of symmetry parallel to the structural frames; while the structural framing that is considered retains the design variations shown in the drawings, all framing on the other side of the symmetry plane is assumed identical. The half of the building with unequal bays at the short face was selected for this study. Second, windows and doors and their associated framing members are neglected for simplicity; girts and metal panels that would normally be interrupted to accommodate openings are simply left intact. If windows or doors were left open, or if they were to fail early during the blast application, the exterior and interior pressures due to leakage pressure into the building would reduce the net loads on the structure. Assuming a sealed building envelope, however, is more consistent with current evaluation methods.

\section{Analysis Descriptions}

Five separate analyses were performed on the structure, with each successive analysis employing a higher level of analytical sophistication and refinement over the previous analysis. For each analysis, the damage incurred by various structural components was assessed and compared against other analyses. The individual analyses are described below.

In all analyses, the building was subject to incident blast loads with peak pressures of 0.7 psi and peak impulse of 20 psi-ms. The long side of the structure was assumed oriented toward the blast source, making it subject to reflected loads.

For analyses using prescribed loads, loading functions were chosen to be consistent with commonly used design guides. Specifically, a standard shock load with a triangular pulse was assumed at all faces. The side faces, rear face, and roof were all subject to incident loads, while the front face was subject to reflected loads equal to twice the incident loads, or 1.4 psi and 40 psi-ms. 


\section{Analysis 1: SDOF Analysis of Components}

The structure was first analyzed component-by-component using standard SDOF methods. In this approach, each component is considered to behave as a simple spring-supported mass. A resistance-displacement curve is defined for the spring to reflect the flexural capacity of the component. In the analyses performed here, bilinear resistance-displacement curves were used to represent the elasto-plastic response of the components in bending.

Each spring-mass system is subject to a loading function defined as the blast pressure time history multiplied by the tributary area of the component. The loading functions at each face followed the prescribed loads described above.

For each component, a displacement response history is computed for the applied loads. Displacements are tracked at the midspan of the component. The peak displacements at midspan are used to compute the equivalent rotations at the member ends. These rotations are determined based on a straight line approximation between the support end and the midspan as shown in Figure 1.

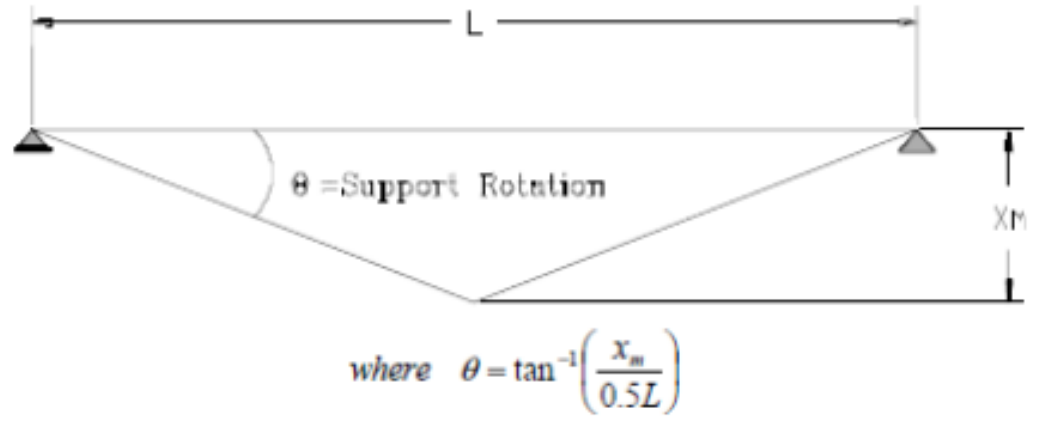

Figure 1: SDOF calculation of component end rotation

These end rotations, in conjunction with measures of ductility, serve as measures of component damage, with various building codes and standards correlating end rotations and ductilities to levels of "Low," "Moderate," and "Heavy" damage.

All SDOF analyses were conducted using SBEDS, ${ }^{1}$ a widely used SDOF analysis tool sponsored by the US Army Corps of Engineers, Protective Design Center.

\section{Analysis 2: Finite Element Analysis of Components and Partial Structures}

As a first level of refinement, SDOF analysis was replaced by dynamic finite element simulation. In this approach, finite element models of individual components and partial assemblages of components are constructed and analyzed for dynamic loading. In contrast to SDOF methods, finite element methods have the potential to capture the response of the structure in far greater detail. With proper modeling, such analysis can accurately capture multiple simultaneous response modes in multiple degrees of freedom, including bending, shear, axial forces, and localized or global buckling. The inclusion of sophisticated material damage models allows the

\footnotetext{
${ }^{1}$ SBEDS [ver.5.0 Dec.2012], Single degree of freedom Blast Effects Design Spreadsheet
} 
model to capture localized damage and yielding. In assemblages, contact algorithms capture the interactions between various components. Examples of single component and assemblage models and the level of detailed damage they are capable of capturing are shown in Figure 2 through Figure 4.

Throughout this study, finite element analyses were performed using NLFlex (Vaughan, 1983). Developed by Thornton Tomasetti's Weidlinger Applied Science division (TT/WAS,) NLFlex is a state-of-the-art, general-purpose, nonlinear, high-fidelity physics-based code. Throughout its $30+$ years of use, it has been repeatedly validated and successfully used to simulate the blast response of structures. Its core solver uses a second order, central difference, explicit time integration approach to compute the dynamic response of structures subjected to extreme loads. NLFlex includes both geometric and material nonlinearities, the latter through the use of sophisticated constitutive models. Nonlinear material effects that are important for blast modeling such as rate effects, post-yield hardening, ductile failure, and post-peak material softening are well represented. NLFlex has been validated for analysis of metal structures subjected to blast and impact loading since the 1990's.

In this set of analyses, the prescribed triangular shock loads described above are used.

While the members in this series of analyses were generally modeled with high fidelity, some modeling simplifications were made. Specifically, supports and restraints were modeled as idealized conditions. Models of assemblages of components utilized symmetry planes to reduce model size and analysis time. Also, connections of girts and purlins to structural frames were modeled as fully bonded at their web (girts) or flange (purlins), or effectively welded; bolted and screwed connections were not explicitly modeled. The inclusion of detailed connections could serve as an additional level of refinement in future studies but was not considered relevant to the objectives in this paper.

Figure 2 - Finite element model of an individual PEMB girt 


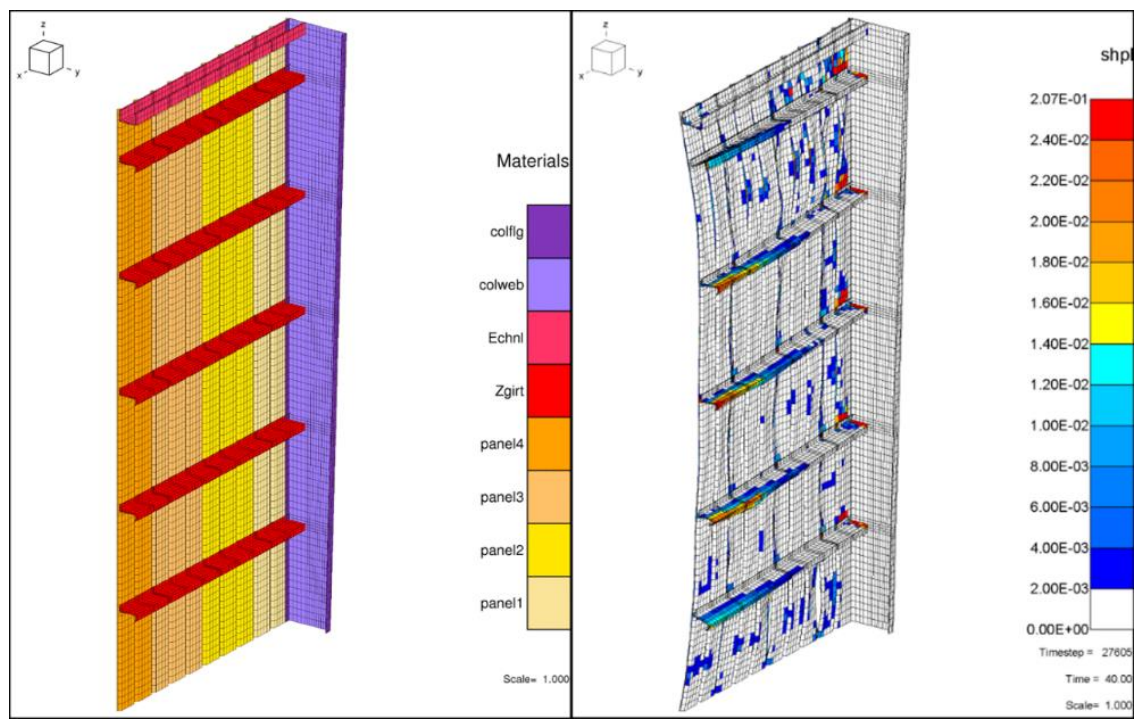

Figure 3 - Typical finite element model of a single wall bay assemblage utilizing symmetry planes at the column line and mid-bay. The left image shows a plot of the full assemblage model. The right image shows localized plastic deformations that develop at girt midspans and supports when subject to exterior blast loads.

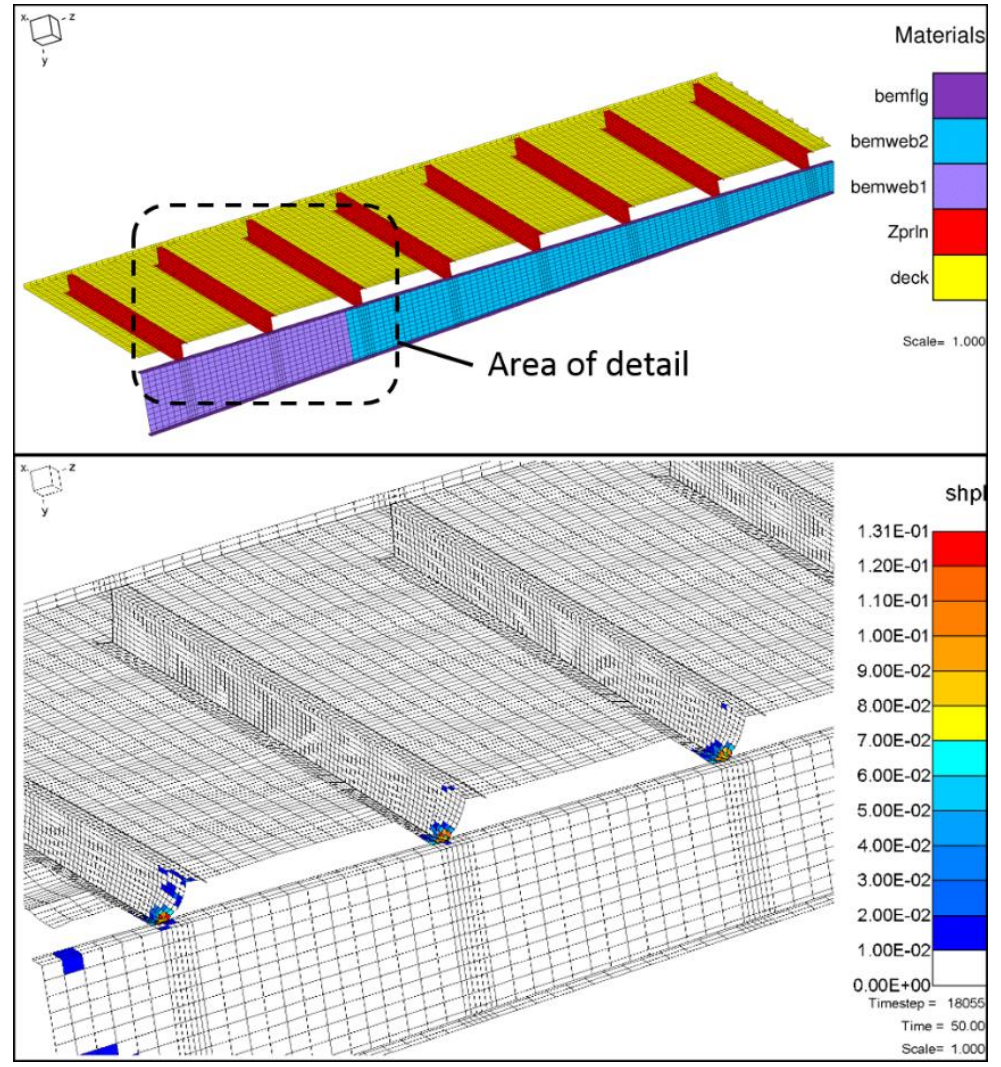

Figure 4-Typical finite element model of a roof bay assemblage utilizing symmetry planes at the column line and mid-bay. The upper image shows a plot of the full assemblage model. The lower image shows localized plastic deformations that develop at the bases of the purlins when subject to blast loads from above. 


\section{Analysis 3: Finite Element Analysis of the Entire Structure}

The next level of refinement involves modeling the entire building as a cohesive system. This approach is effectively an extension of the assemblage models of Analysis 2. In considering the complete structural system, the model is able to account for global building behaviors, such as the differential motions of the steel frames. The stiffening effects of purlin continuity across frame supports is captured. The inclusion of details such as purlin straps can help distribute load and stiffen the structure. It also includes the stiffening effects of interacting components, such as potential composite action between metal panels and girts and purlins, as discussed above.

Another advantage of the larger model is that it provides more realistic support conditions of virtually every component in the model. For example, supports of individual girts and purlins in the prior analyses were considered rigid. With the full building model, girts and purlins are attached to steel frames which deflect and sway in response to the blast loads. Rather than rigid, the girt and purlin supports are effectively softened, which may reduce the degree of damage they incur. In this model, the bases of the steel frames and end columns are modeled as idealized fixed, but assuming the base connections do not fail, this is a reasonable assumption.

Plots of the finite element model are shown in Figure 5 through Figure 8. In order to speed analysis, the building is modeled with a plane of symmetry through the center bay, as described above.

In this model, the prescribed loads described above are applied as pressure histories directly to the exterior metal panel cladding. While a triangular load function is still assumed, the load is swept across the building according to established shock propagation speeds. Whereas SDOF analyses assume the load is applied uniformly across a component, this analyses considers loads that effectively travel along members, such as girts at the building's side faces and the roof beams in the structural frames. This gives a more realistic response in these members compared to SDOF analysis. Similarly, the phasing of loads between the front and rear faces should provide a more realistic assessment of building sway of the moment frames. The applied loads are shown in Figure 9. 


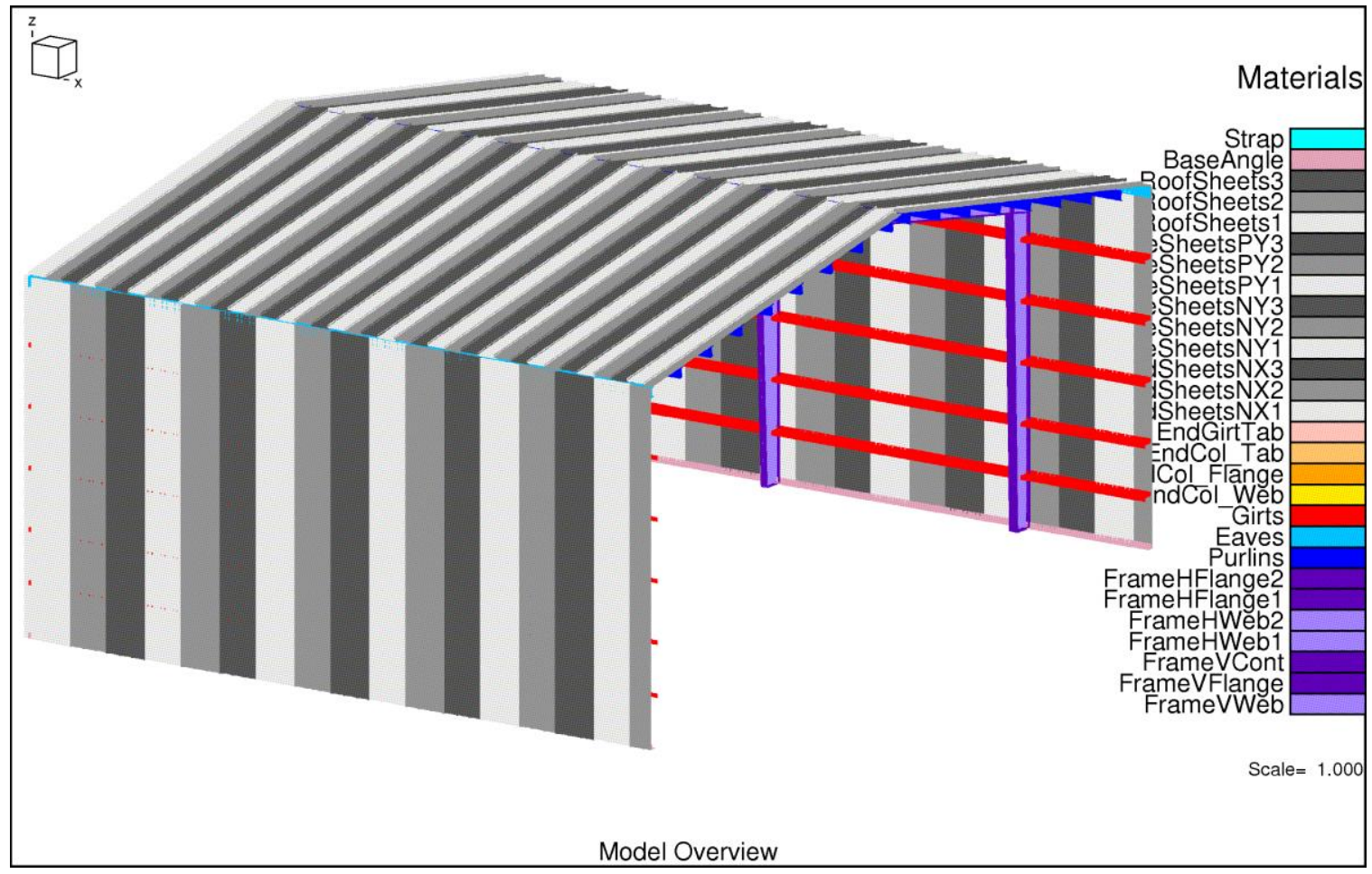

Figure 5 - Overview of the full building finite element model. The model employs a symmetry plane through the center of the structure. Varying shades of grey are used to highlight individual metal panels.

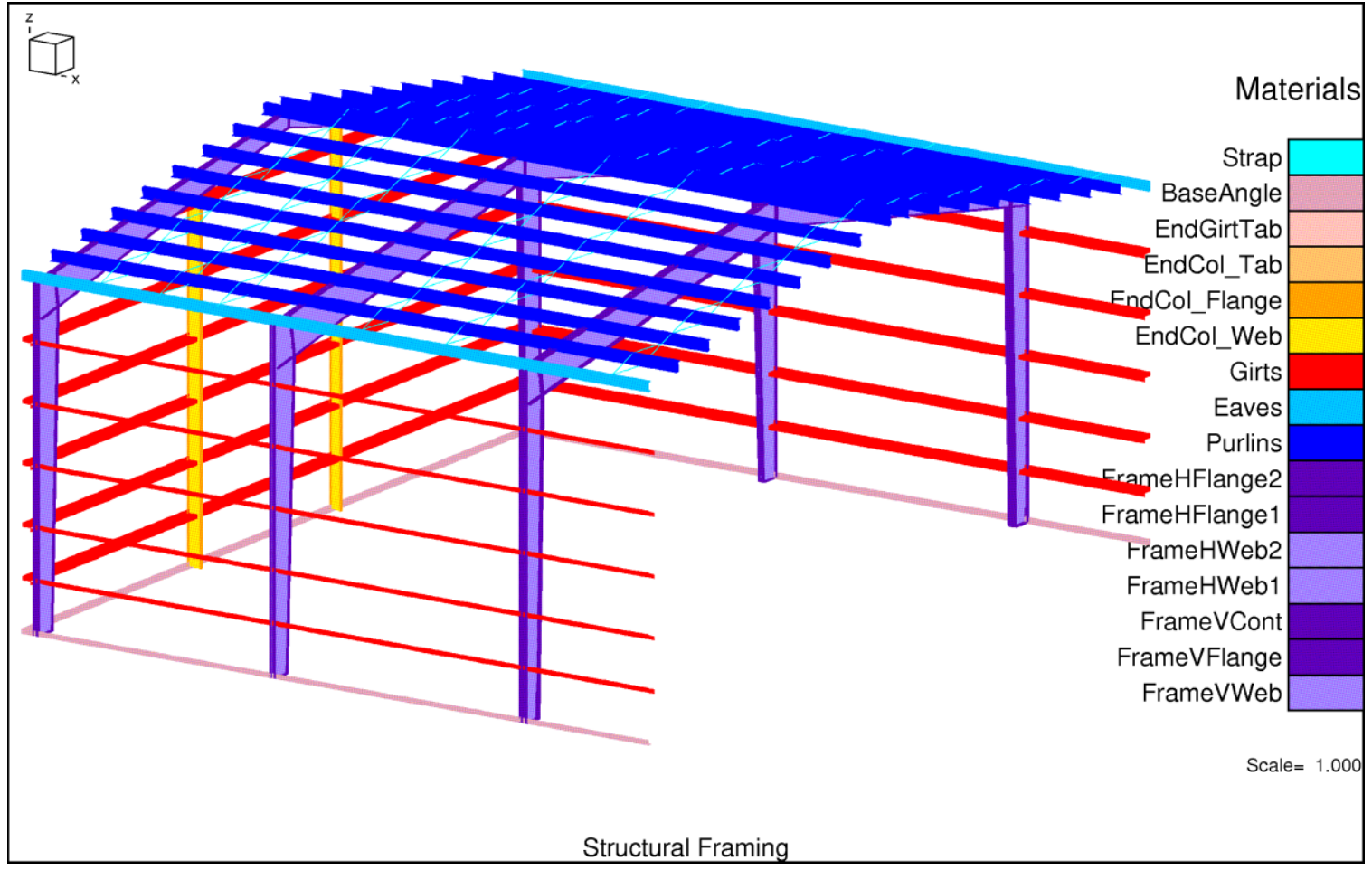

Figure 6 - Steel framing. The model employs a symmetry plane through the center of the structure. 


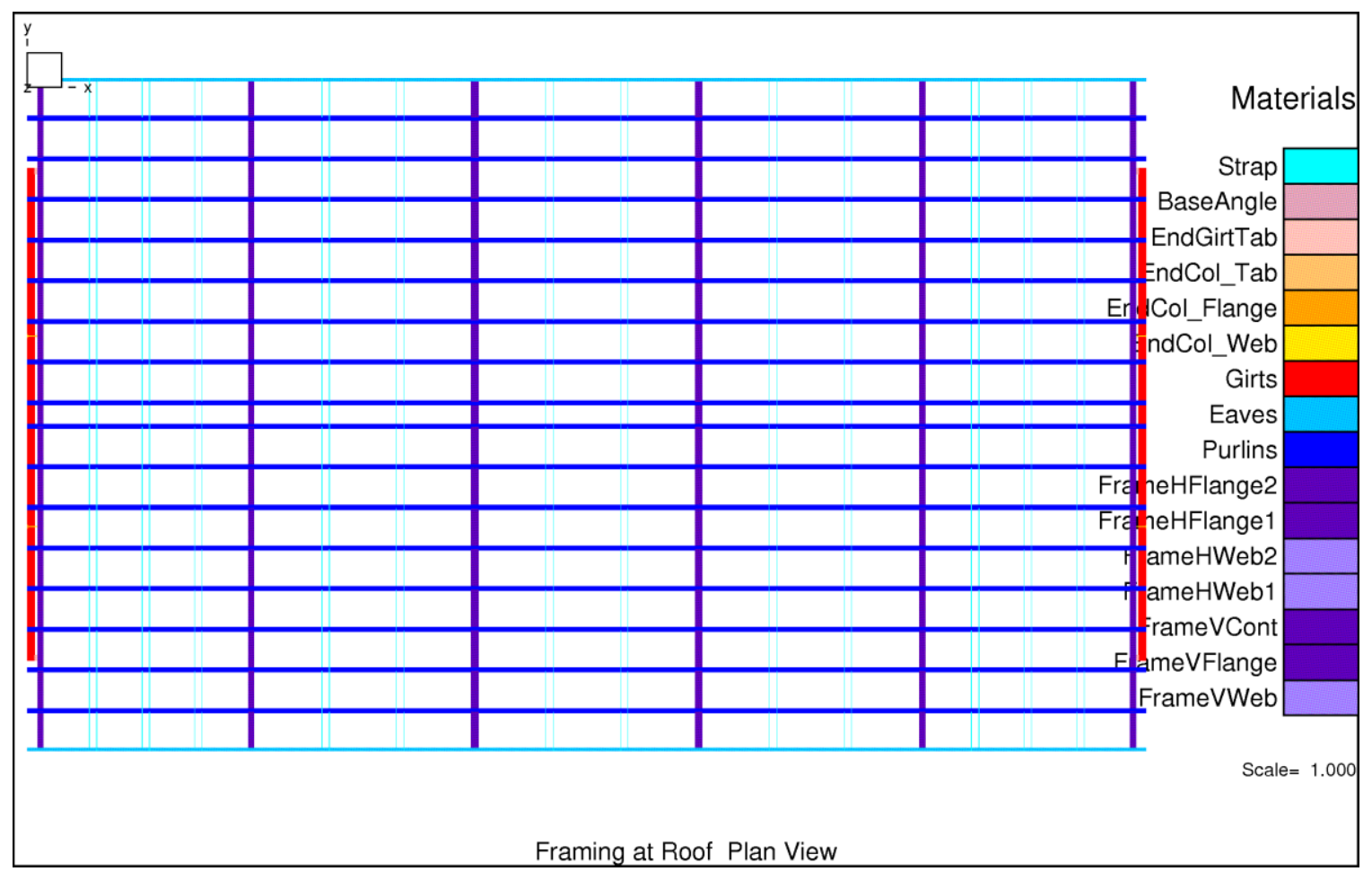

Figure 7 -Roof framing. While the model employs a symmetry plane through the center of the building, the structure here is mirrored across that plane to show the full building roof.

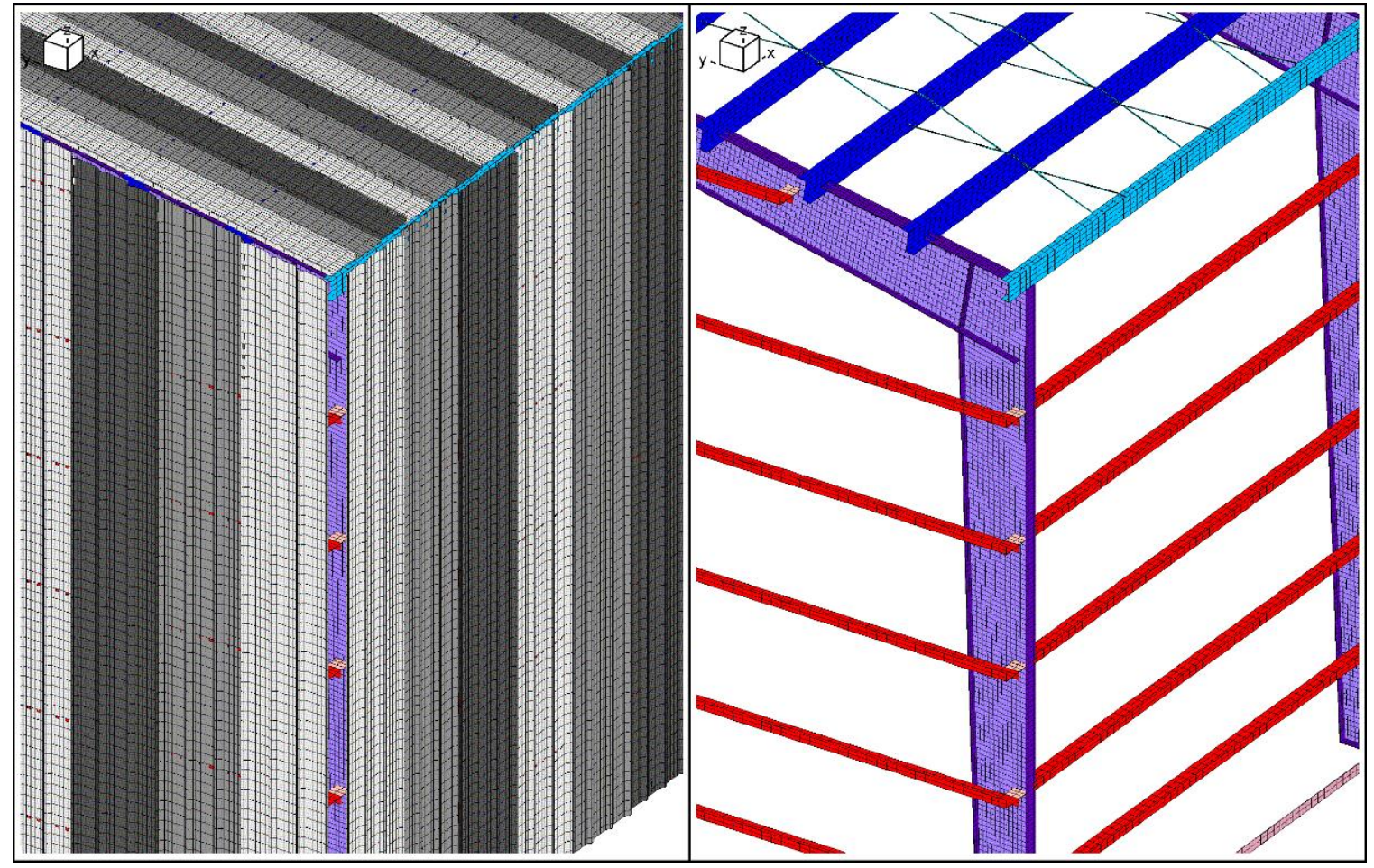

Figure 8 -Framing details at the building corner. Varying shades of grey are used to highlight individual metal panels. 


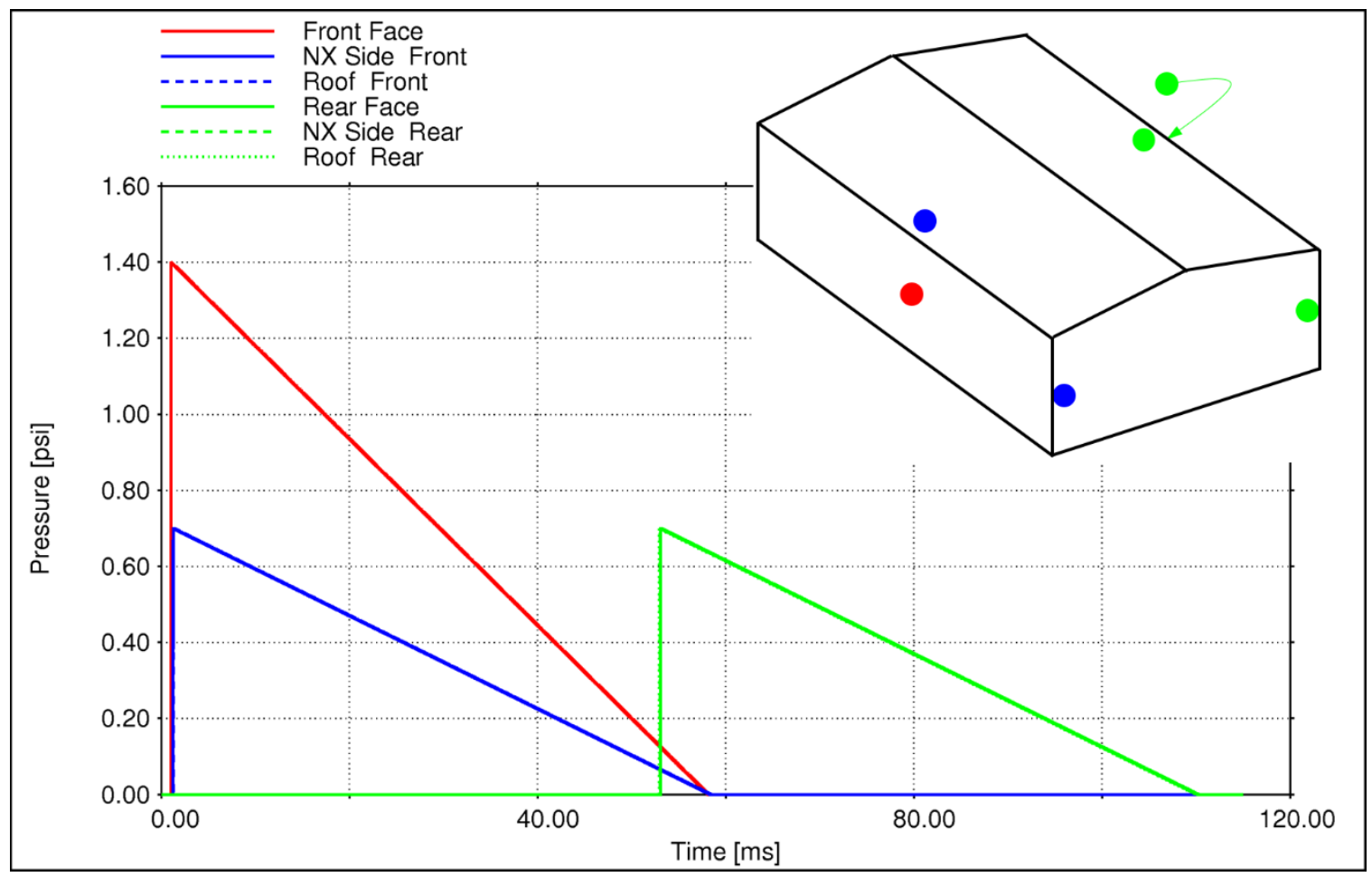

Figure 9-Applied loading in Analysis 3 and 4

\section{Analysis 4: Finite Element Analysis Including Internal Air Pressure}

The next level of refinement includes the effects of internal air pressure on the structural response. This is achieved by coupling the structural finite element analysis with a simultaneous computational fluid dynamics (CFD) analysis. As the external blast pressure causes the building surfaces to deform inward, the motion of those surfaces is resisted by internal air pressure; just as flowing wind exerts a pressure force on a structure, a structure moving through air will meet similar resistance. In other words, PEMB's tend to be light framed buildings having little inertial resistance and are very flexible. Therefore, PEBM's can incur large deflections during the application, or duration of the blast load that can be resisted by internal shocked air pressure. This "tent" effect can improve the capacity of the PEMB structural framing.

Internal air pressures are computed with VCFD, a finite volume CFD code developed by TT/WAS to solve the inviscid compressible Euler equations on a fixed Cartesian mesh. It includes a simplified vapor cloud source as described in (Wesevich, 2016,) making it particularly suitable for the analyses conducted here.

VCFD implements the MUSCL-Hancock method with "total variation diminishing" (TVD) conditions to provide second-order accurate solutions in space and time. For the modeling of blast propagation, this method produces accurate pressure waveforms at far field locations. For such scenarios, the VCFD computational strategy is to define and run a carefully selected series of expanding grids that capture the deflagration or detonation and the propagation of their resulting pressure waves. For example, a VCE calculation can be run in 1D spherical geometry until just before the blast wave impacts the first structure. Subsequent time-integration in 2D/3D 
are very efficient, as VCFD currently supports symmetric multi-processing (SMP) parallel computations using OpenMP directives that fully utilize the multi-core multi-CPU resources available on a single computer.

VCFD was initially developed for simulating high explosive detonation effects on military and civilian structures and is fully coupled with NLFlex. It has been verified by comparisons to analytical shock solutions and code-to-code comparisons with other CFD software. It has been validated against many field and lab scale tests, including cased and embedded munitions as well as bare charges. Of particular interest, it has compared well to field tests for reflected pressures from large charges at very large standoff, and pressures on the back sides of structures in simulated cities which exhibit channeling and shielding effects. Wesevich (2016) also validates VCFD simulations against vapor cloud deflagration experiments. This leads to confidence in the ability of VCFD to accurately propagate blast waves and predict their interactions with structures and as equally important, account for shielding and channeling effects due to obstructions in front of the building of interest.

In this analysis, CFD is used to compute the internal pressure loads only. The same sweeping triangular pulse loads described above are applied to the building exterior, while any external air pressures resulting from building deformations are not applied to the structure. With no windows or doors that can break, the only mechanism for venting and relief of internal pressure is loss of cladding. The model also includes no internal partitions that may serve to increase the effects of internal pressure.

\section{Analysis 5: Finite Element Analysis Including Fully Coupled VCE Loads}

The final analysis replaces the sweeping triangular pulse loads with loads derived from a fully coupled CFD analysis of a vapor cloud explosion (VCE.) In contrast to analysis 4, both internal and external air pressures applied to the building are taken from the CFD analysis.

Using a trial an error approach, a vapor cloud size and center were determined that resulted in incident pressures of approximately 0.7 psi with a 20 psi-ms impulse, similar to that used in all prior analyses. Reflected pressures and clearing effects were directly computed internally by the CFD code based on first principle numeric algorithms. Rather than a triangular pulse, the resulting pressure history is smoother and less shock-like, which is representative of typical VCE loads (Wesevich, 2016). The increased rise time enhances clearing effects, resulting in lower reflected pressure and impulse over much of the front face.

\section{Analysis Results}

The goal of the study is to compare estimates of building damage determined by the various analysis approaches outlined above. This requires a common approach to measuring damage. While the finite element analyses are capable of capturing a variety of damage mechanisms in great detail, as shown in Figure 3 and Figure 4, the information derived from SDOF analysis is limited to parameters such as end rotation and ductility. To facilitate comparison of damage across both SDOF and finite element analyses, end rotations of flexural members are used as a general measure of damage in all analyses instead of levels of plasticity and buckling modes that 
can be captured and quantified in CSD computations. The structural steel frames exhibited very limited damage in all analyses and do not warrant discussion, while damage to the metal panels is not presented here for brevity. Presented results are therefore limited to damage due to inward deflections of girts and purlins; outward deflections due to rebound or negative air pressures are also not presented for brevity.

In order to illustrate the performance of all girts and purlins in the entire structure, results are plotted on charts showing the number of components exceeding the plotted end rotations. Each chart is created by tabulating the end rotations of all members in each analysis and sorting the results from smallest end rotation to largest. The numbers of components exceeding each computed end rotation are then summed and plotted. The result is a plot of cumulative damage that effectively shows the distribution of magnitudes of damage across a specified face of the structure. The resulting charts are shown in Figure 10 through Figure 13. Each chart shows the end rotations associated with peak inward displacements of members grouped by structural face.

SDOF analyses consistently produced the largest end rotations on all faces. While such analyses are generally understood to be conservative, Figure 10 through Figure 13 quantify just how much larger SDOF damage predictions can be compared to finite element results. The conservatism results from the number of simplifications and assumptions either required or customarily used in these analyses, including the assumption of rigid supports, the use of perfectly plastic materials with no strain hardening, the simplification of load rate effects, neglecting the effects of stiffening due to composite action with attached members, inability to capture the actual load path of a multiple degree of freedom system, and the inability to distribute damage. Each of these simplifications and idealizations adds to the degree of conservatism in results.

Analysis 2 was initially conceived to be finite element analyses of individual components, i.e., single girts, purlins, etc., in the manner of SDOF analysis. However, this was quickly seen to be an unworkable approach. As discussed above, the finite element models introduced new mechanisms into the structural response, such as torsional buckling and web buckling. As single component models, however, they lacked any of the attached components that serve to limit or restrain some of these new response mechanisms. These models were therefore prone to unrealistic failures. For example, because the center of load applied to the girts and purlins is not aligned with the shear center of the Z-shaped cross-section, the members tend to twist when loaded along a flange. While this behavior is both realistic and expected, the single component models excluded restraints that would be provided by other structural components such as exterior cladding. As a result, unrealistic degrees of torsional buckling were seen in some single component girt and purlin analyses.

A second example involved localized web buckling. In the early phases of this study, finite element analyses of individual components were conducted for loads other than those presented here. In these preliminary analyses, localized buckling of the webs of girts and purlins was observed for triangular pulse loads with high pressures but low impulses. After some investigation, it was concluded that this buckling resulted from overly conservative assumptions of load application. In modeling the single component, all load from the member's tributary area was applied directly to the girt or purlin in a manner consistent with SDOF analysis. In a real 
structure, however, loads are applied to compliant metal panels which collect and transfer the load to the supporting girt or purlin. In this process, deformations in the panels effectively transform the load function into one of lower pressure but longer duration while preserving the total impulse. This reduction in load intensity is sufficient to prevent unrealistically created localized web buckling.

To prevent the unrealistic responses observed in the individual component models, the models were expanded into small assemblages of components, with each assemblage representing one bay of structure. Typical wall and roof models are shown in Figure 3 and Figure 4. By modeling a more complete, if still limited, system, additional mechanisms were introduced which prevented some of the unrealistic responses seen in the single component analysis. This is demonstrated in Figure 14, in which excessive torsional rotations of girts are shown to be prevented by the attached cladding. These models were seen to be effective and provided results generally in line with those of the other finite element analyses presented here. Given the similarity of results to Analysis 3, 4, and 5, they are omitted from the damage charts for clarity.

Analyses 3, 4, and 5 all show significant improvements in predicted performance compared to SDOF analysis. Improvements are particularly dramatic at the front face, which is subject to reflected pressures and thus sustains the greatest damage. This is shown in Figure 10. With computed end rotations of most members in excess of $25 \mathrm{deg}$, SDOF analysis predicts the failure of virtually all girts on the front face. In contrast, the largest end rotation in any member computed by finite element anlaysis of the full structure is only $10.5 \mathrm{deg}$. Further improvements are achieved with the inclusion of internal pressures, which show the largest peak end rotation as $5.7 \mathrm{deg}$. The inclusion of fully coupled VCE loading shows further performance gains, with the largest peak rotation being only $3.4 \mathrm{deg}$. Plots of peak lateral displacement computed by finite element analysis, also shown in Figure 10, reflect the trends in damage estimates shown in the accompanying chart.

Figure 15 and Figure 16 show the differential internal and external pressures computed near the front face for Analysis 5 with fully coupled VCE loads. Figure 15 shows a snapshot of the CFD analysis. As the blast reflects off the front face of the structure, it generates a compression wave that propagates throughout the building interior. This snapshot shows the development of increased pressures at the interior of the front face as it deflects inward.

Figure 16 shows time histories of pressure recorded near the interior and exterior faces of the front and rear walls; these histories were recorded at points offset from the building face and are therefore slightly lower than the actual reflected pressures applied to the surfaces. Near the front face, peak external calculated pressures of 1.15 psi are partially cancelled by peak internal calculated pressures of $0.25 \mathrm{psi}$, resulting in a net pressure of only $0.9 \mathrm{psi}$. This effectively shows that internal pressures do indeed offset external pressures, resulting in a lower net load. Though results in this figure are for Analysis 5, loads at the front face are similarly reduced in Analysis 4, which considers prescribed triangular pulse loading with coupled internal pressure.

Figure 16 through Figure 18 show the actual reflected pressures and impulses applied to the structure in Analysis 5. Together, they show that further performance improvements seen in that 
analysis are due to the lower reflected pressures computed by CFD. Analysis 1 thru 4 assumed reflected pressures and impulse at the front face to be equal to twice the incident pressure, consistent with typical design codes. In this case, however, CFD analysis produces peak reflected pressures only $65 \%$ higher than incident, and peak impulse about $80 \%$ higher.

Additional features seen in Figure 17 are the sinusoidal nature of the load waveform computed by CFD, with a non-zero rise time and a significant phase of negative loading. Both features may also result in smaller inward girt displacements, though the calculated negative phase of loading also increases outward displacements.

At the side faces, Figure 11 shows that all three finite element analyses predict significantly better performance over SDOF analysis. While the inclusion of internal air pressure predicts somewhat better performance than analysis without, further refinement to include VCE loads produces negligible gain. While the CFD loads at the side faces of Analysis 5 are slightly lower than the prescribed loads of Analysis 4, the difference is small, about 0.65 psi and 17.5 psi-ms vs. 0.7 psi and 20.0 psi-ms. Furthermore, while Figure 17 shows that peak CFD pressures decay as they travel down the side of the building, peak impulses show less decay. Plots of peak lateral displacement of the side faces including W10 and W12 columns[MD1], also shown in Figure 11, reflect the general trends in damage estimates shown in the accompanying chart. At first glance, these plots suggest that the analysis with prescribed loads and internal pressure should produce more girt damage relative to the analysis with coupled VCE loads than is suggested by the accompanying damage plots. However, damage is computed by considering the midpoint displacement relative to end support displacement. At the side walls, the intermediate W10 and W12 columns are deforming; the attached girts therefore have significant end displacements which are considered in calculating their end rotations.

At the rear face, Figure 12 also shows that all three finite element analyses predict significantly better performance over SDOF analysis. In contrast to the side faces, inclusion of internal air pressure does not appear to contribute any appreciable improvement in performance. The use of fully coupled CFD loads, however, does provide further improvement. As shown in Figure 17 and Figure 18, this is due to lower applied loads at the rear face. While Analyses 3 and 4 apply incident loads as typically specified by design codes, CFD analysis produces reduced loads of 0.4 psi and 13.5 psi-ms. These peak pressures are about $43 \%$ lower than those using prescribed loads, while peak impulse is about $35 \%$ lower. Plots of peak lateral displacement of the moment frames, also shown in Figure 12, mostly reflect the general trends in damage estimates shown in the accompanying chart. Similar to plots at the side face, plots of peak inward displacement for analysis with prescribed loads and no internal pressure are not indicative of damage due to motions of the moment frames.

Consistent with all other faces, Figure 13 shows that all three finite element analyses predict better performance of the roof purlins than SDOF analysis. While Analyses 3 and 4 produce nearly identical damage assessments, the results of Analysis 4 with prescribed loads and internal air pressure are an outlier. In this analysis, inward deformation of the front and side faces increases the internal air pressure near those faces. This creates an upward deflection in the end spans of the continuous purlins, resulting in the lower computed end rotations due to downward 
displacement shown in the plots. This analysis case exhibits generally larger peak upward deflections than the other cases.
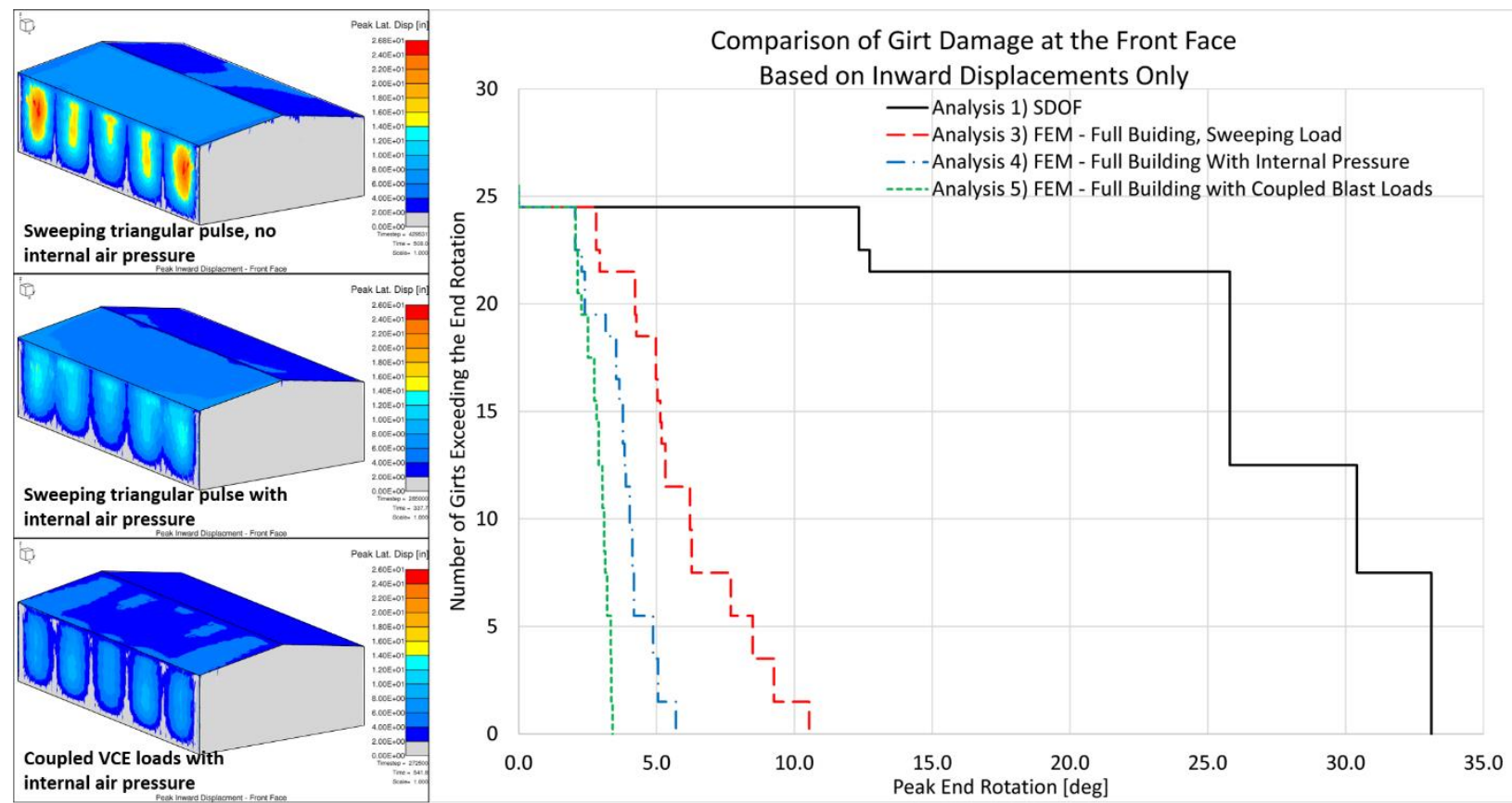

Figure 10 - Plots of cumulative damage and peak lateral displacement at the front face
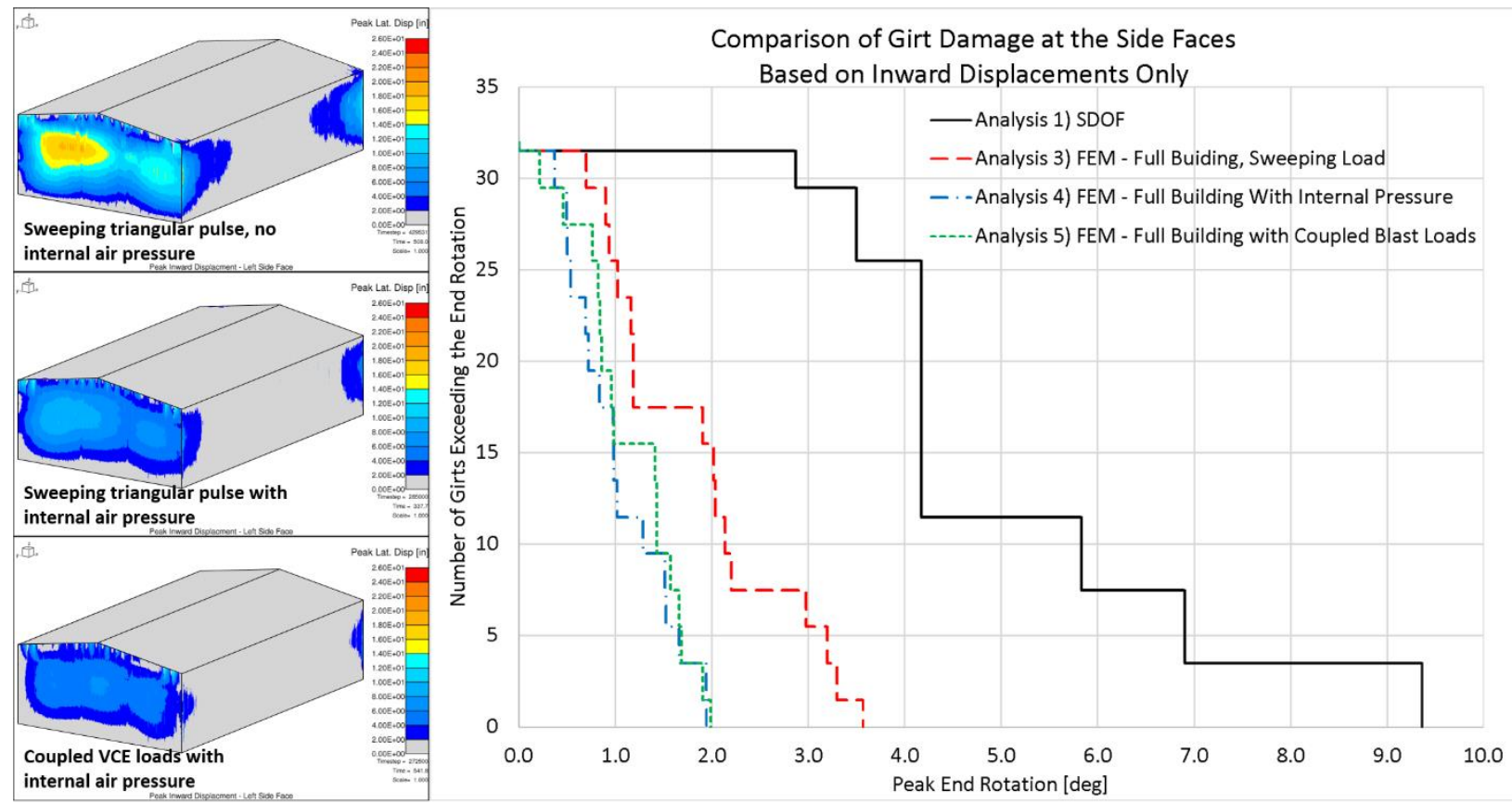

Figure 11 - Plots of cumulative damage and peak lateral displacement at the side face 

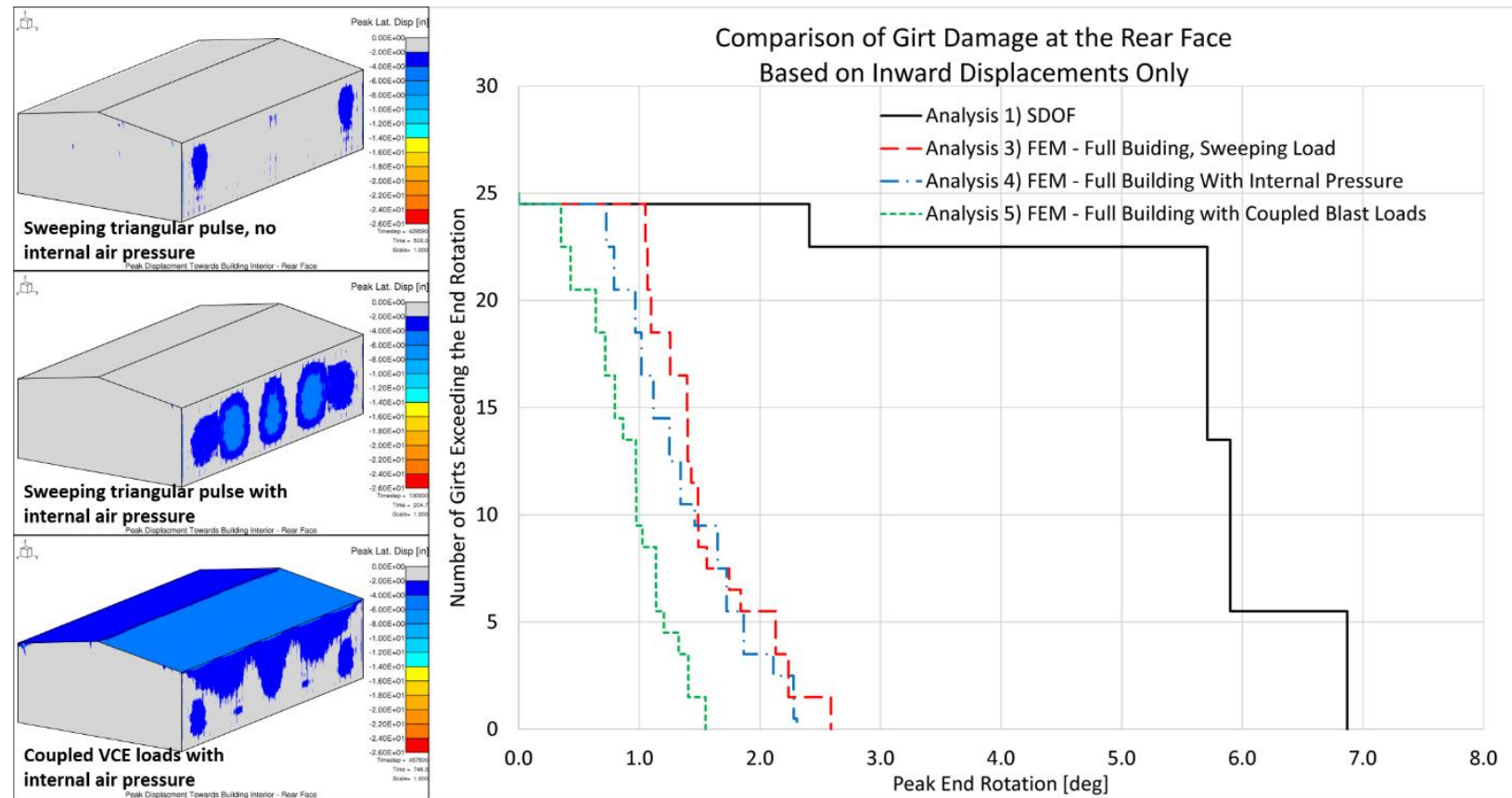

Figure 12 - Plots of cumulative damage and peak lateral displacement at the rear face
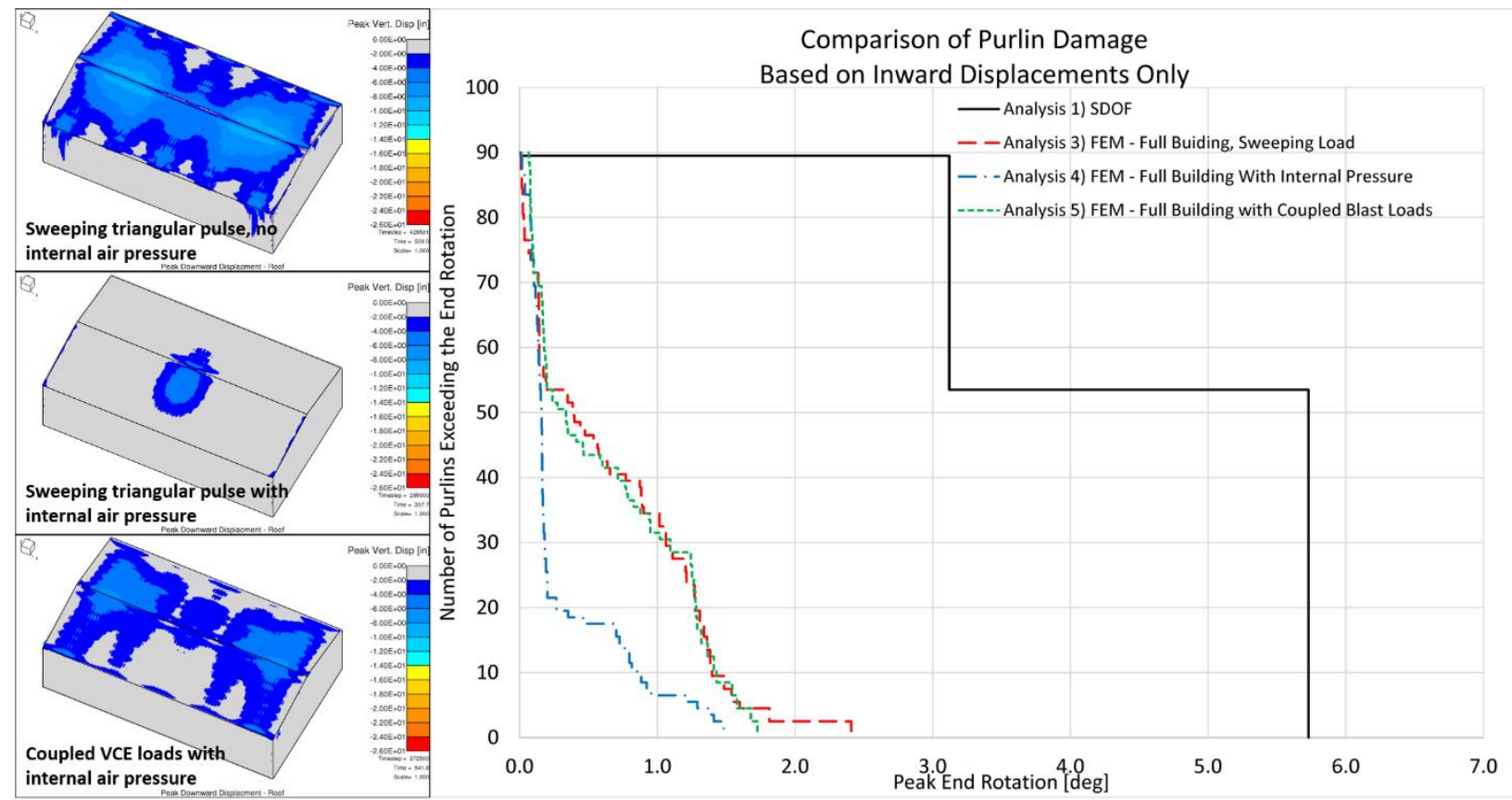

Figure 13 - Plots of cumulative damage and peak downward displacement at the roof 


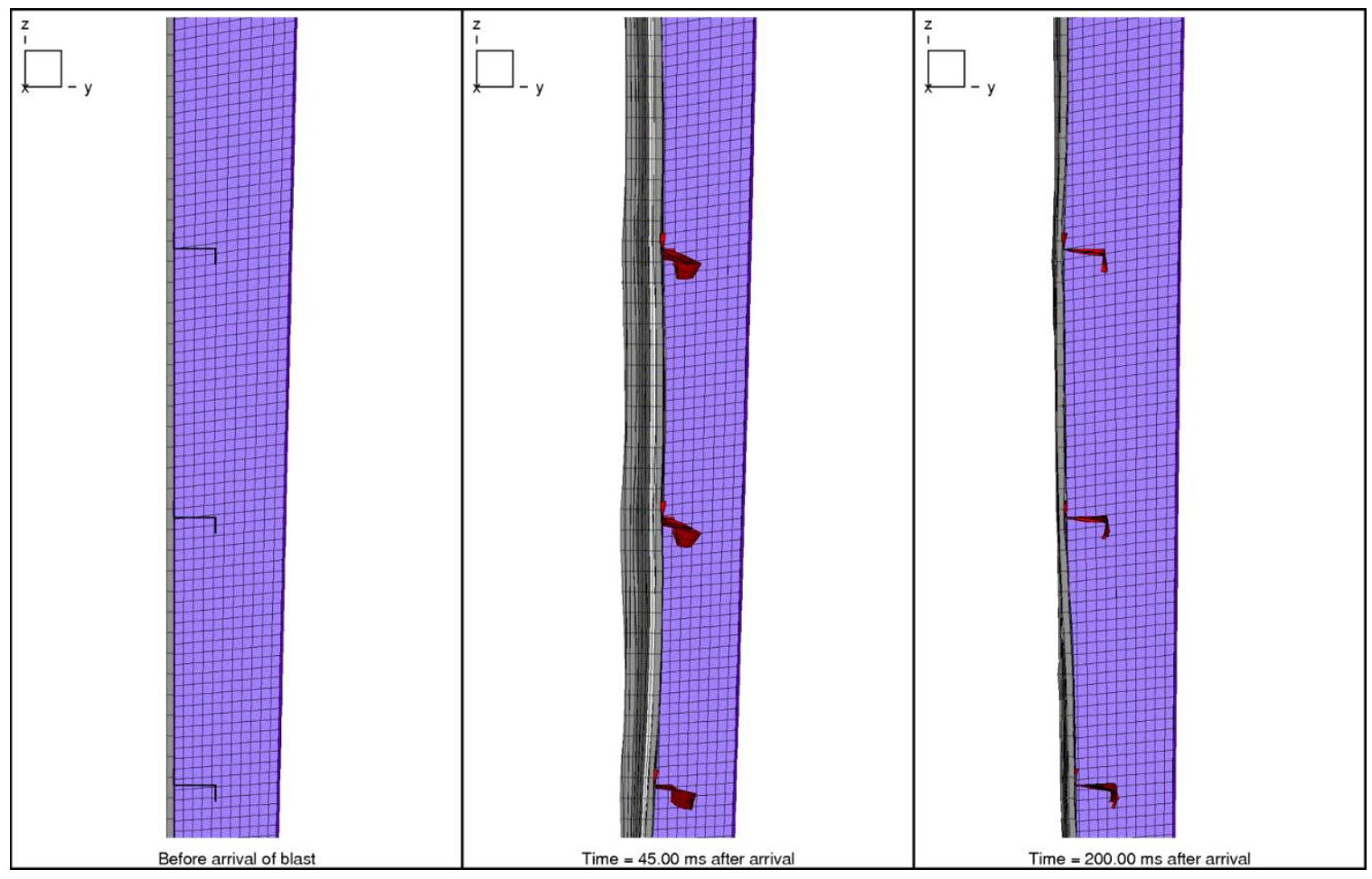

Figure 14 - Cross-section through the front face. As the front face deflects inward, the girts twist due to eccentricities between the center of load and the shear center of the Z-shaped cross-sections. Though the torsional rotations are large, the attached metal panel cladding restrains the girts, allowing them to rebound.

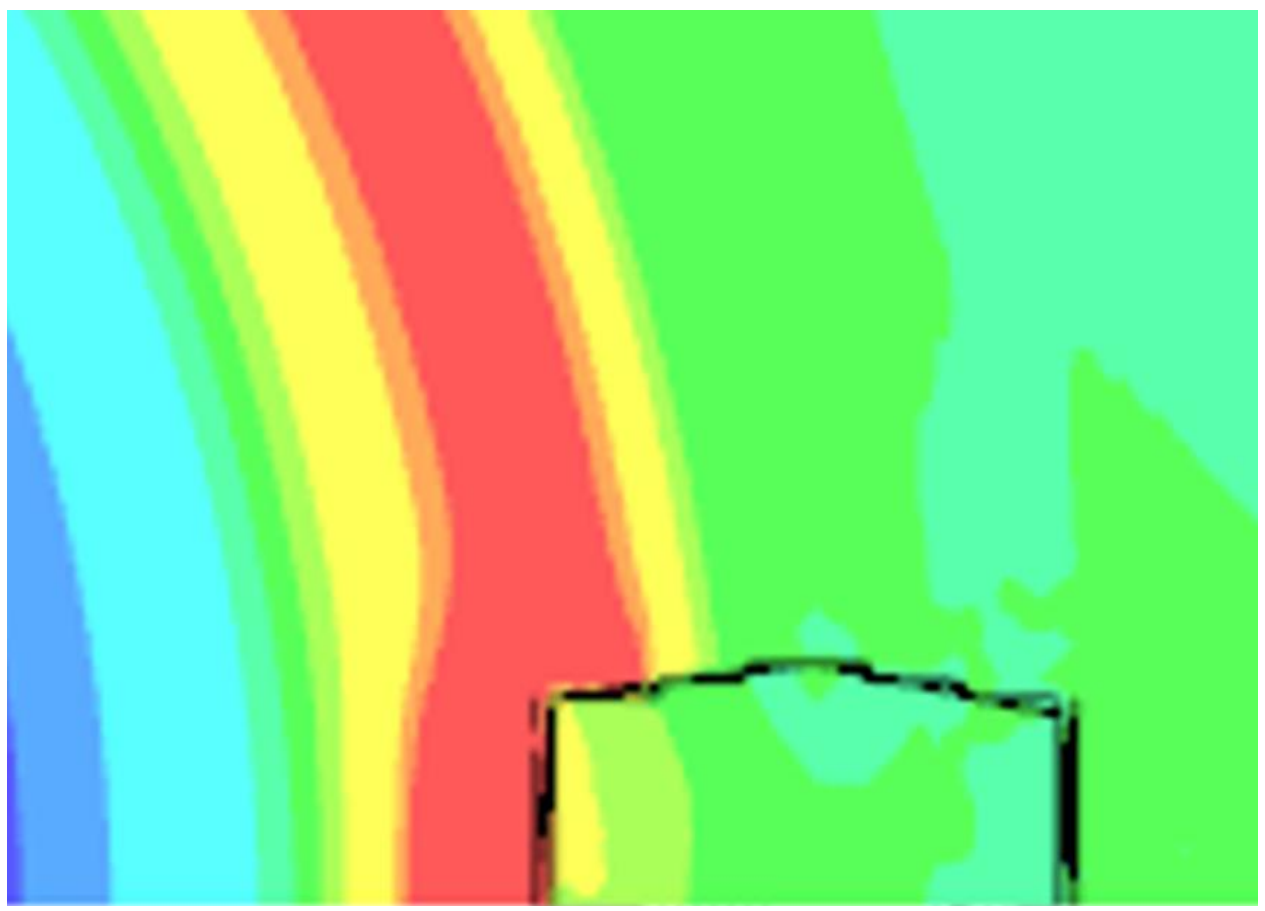

Figure 15 - Cross-section of the CFD analysis. As the blast front reflects off the front face of the building, the structure deforms inward, creating waves of lower intensity that propagate through the building interior. 


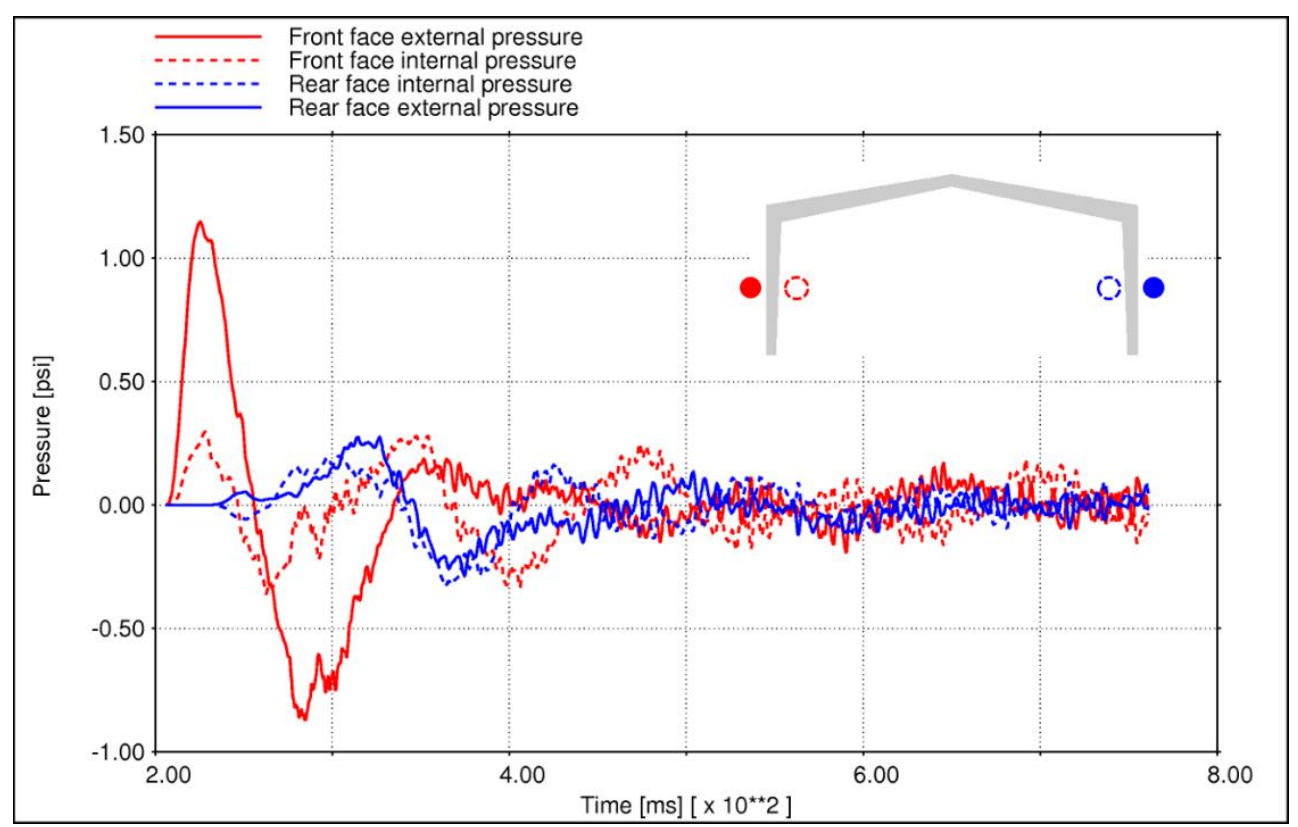

Figure 16 - Differential internal and external pressures for analysis 5 with fully coupled VCE loads. Near the front face, peak interior pressures of 0.25 psi partially mitigate the effects of the 1.15 psi external pressure, resulting in a pressure differential of only about 0.9 psi. Note that these pressures are measured at points offset from the structure and thus do not represent actual applied, reflected loads on the structure. The pressure differential at the rear face is less significant.

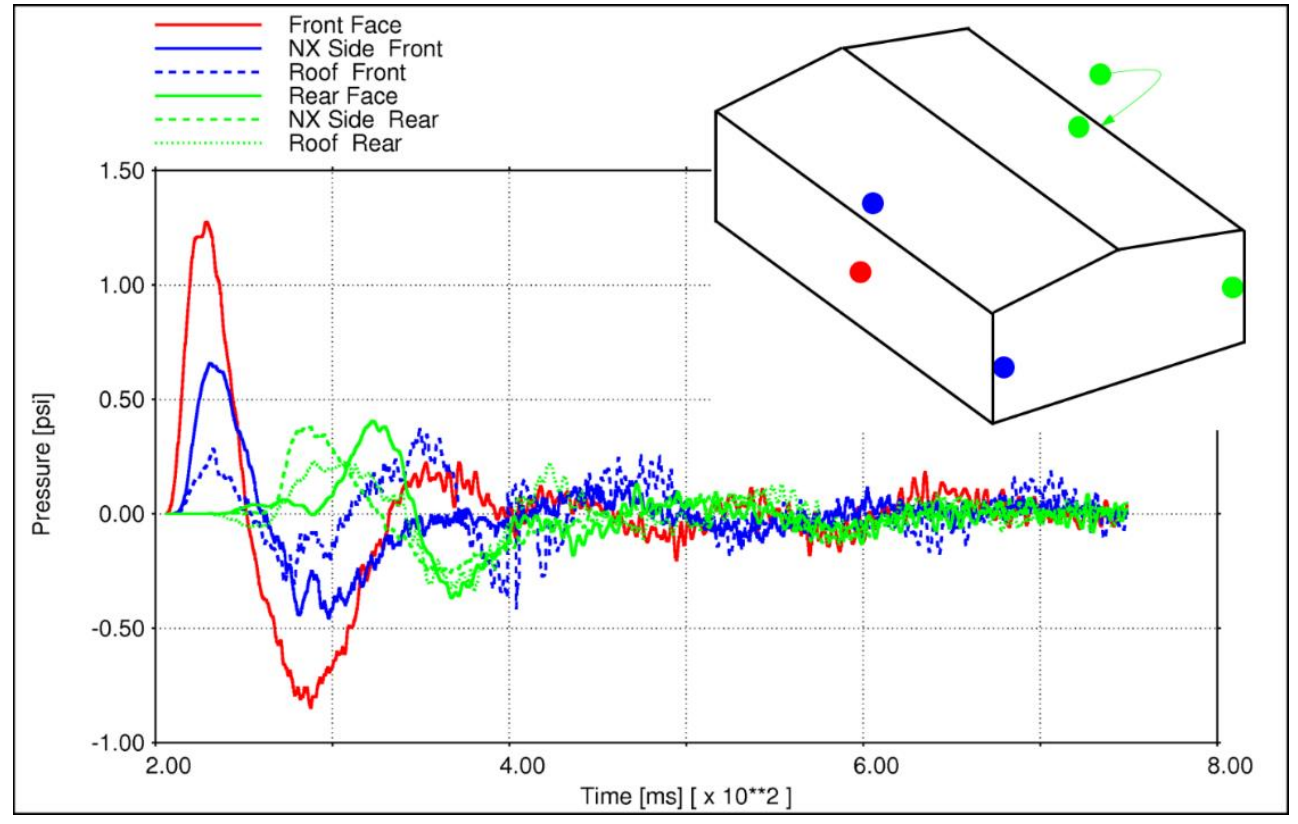

Figure 17 - External pressure loads computed with a fully coupled CFD analysis using a VCE waveform. Reflected pressures at the front face are lower than would be prescribed by most design codes. Furthermore, the waveform is more sinusoidal than triangular, with a non-zero rise time and a significant phase of negative loading. Pressures decay with distance, resulting in loads at the rear face significantly lower than incident. 


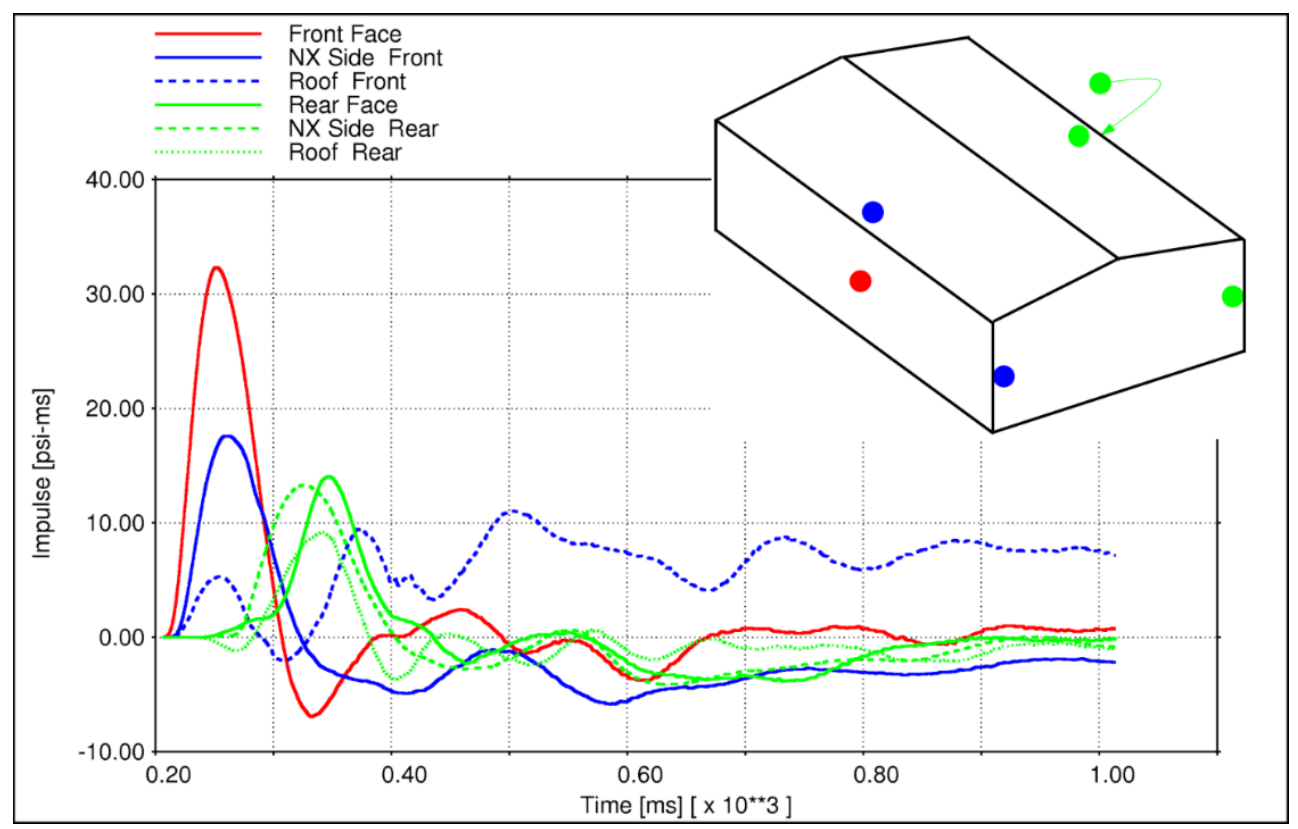

Figure 18 - External impulse histories computed with a fully coupled CFD analysis using a VCE waveform. Impulses due to reflected loads at the front face are lower than would be prescribed by most design codes.

\section{Conclusion}

While SDOF analysis is generally recognized as producing conservative predictions of structural performance of PEMBs subject to vapor cloud explosions, the preceding analyses demonstrate and quantify the extent to which this is true. The use of advanced computational techniques such as finite element and CFD analyses was consistently seen to predict dramatically better performance of the structure. While these findings warrant confirmation with field testing, they are in line with generally acknowledged findings from actual events.

\section{Recomendations}

While detailed finite element analyses may be not be justifiable or even feasible for every PEMB performance assessment, these analyses demonstrate the potential for them to play other valuable roles. For example, they may be used in developing more simplified analysis tools, such as P-I curves of isodamage for common structures. They may be used in the development or refinement of design guidelines, such as determining less conservative end rotation, ductility, or other criteria commonly used when SDOF analysis is required. In any context, they should be effective for any analysis that requires an improved assessment of structural damage, and will thus provide better estimates of occupant vulnerability. It is also recommended that advanced analyses of PEMB's be used for new and existing building blast hardening projects supported by screening level SDOF and semi-empirical based pressure-impulse driven results to comply with consequence or QRA facility siting goals. The cost savings may be substantial in employing the advanced numerical methods described in this paper. 


\section{References}

American Society of Civil Engineers, “ASCE Blast Design of Petrochemical Facilities”, 2010

Center of Chemical Process Safety, American Institute of Chemical Engineeers, Guidlines for Evaluating Process Plant Buildings for External Explosions and Fires, 1996.

Center of Chemical Process Safety, American Institute of Chemical Engineeers, Guidlines for Vapor Cloud Explosion, Pressure Vessel Burst, BLEVE and Flash Fire Hazards, Hoboken, NJ : John Wiley \& Sons, $2^{\text {nd }}$ Edition, 2012.

Hassig, P., 2017. VCFD User Manual. Cupertino, CA. Thornton Tomasetti

SBEDS, "Single degree of freedom Blast Effects Design Spreadsheet", Version 5.0, December 2012, Protective Design Center, US Army Corps of Engineers, Omaha, NE (https://pdc.usace.army.mil/software/sbeds)

Vaughan, D.K., “FLEX User's Guide,” Report UG8298, Weidlinger Associates, Mountain View, CA, May 1983 plus updates through 2015.

Wesevich, J., P. Hassig, L. Nikodym, V. Nasri, J. Mould, “Accounting for Channeling and Shielding Effects for Vapor Cloud Explosions," Mark Kay O'Connor Process Safety Center International Symposium, Oct. 25, 2016, College Station, TX. 